\title{
Impact of surgeon's hand and ocular dominance on right and left total knee arthroplasty alignment
}

\author{
Shizar Goosheh, Jacalyn Thoren, Douglas Naudie, Mathew Teeter, James Howard, Brent Lanting
}

Faculty Reviewer: Jacquelyn Marsh, PhD (School of Physical Therapy)

\section{ABSTRACT}

Postoperative alignment is an important modifiable confounder that contributes to the longevity of total knee arthroplasty (TKA). Studies have shown that surgeon's handedness can affect surgical performance; however, no studies have assessed the effect of surgeon's hand or ocular dominance on TKA alignment. The purpose of this study was to evaluate the effect of surgeon's hand and ocular dominance on coronal plane alignment in TKA.

We retrospectively evaluated 138 patients who underwent sequential bilateral TKA by the same surgeon, using the Genesis II PS knee (Smith \& Nephew, Memphis, TN). We assessed postoperative alignment by measuring and comparing anatomical tibiofemoral angle (TFA) bilaterally on standard postoperative knee radiographs, as well as Knee Society function and pain scores to determine any functional differences. Lastly, we evaluated whether a crossed hand-ocular dominant surgeon had greater accuracy when performing a TKA on the side opposite their hand dominance compared to uncrossed hand-ocular dominant surgeons.

All surgeons were right-hand dominant and there was a significantly larger anatomical TFA on left TKAs (mean [SD], 4.6 $\left.\left[2.8^{\circ}\right]\right)$ compared to right TKAs $\left(3.8^{\circ}\left[2.5^{\circ}\right]\right)(\mathrm{P}=0.003)$. There was no significant difference between right and left Knee Society function ( $P=0.09)$ and pain scores $(P=0.86)$. When comparing left TKAs, surgeons with uncrossed hand-ocular dominance $\left(4.5^{\circ}\right)$ performed with equal accuracy compared to surgeons with crossed hand-ocular dominance $\left(4.8^{\circ}\right)(2$-tailed test $=0.597)$, indicating no effect of ocular dominance.

In summary, hand but not ocular dominance was shown to have significant postoperative alignment effects on TKA.

\section{INTRODUCTION}

Total knee arthroplasty (TKA) is a popular procedure for endstage osteoarthritis. It is generally a successful procedure with implant survival approaching 15 years, and with the general population living longer, extended durability of the implant is necessary. One modifiable variable affecting the longevity of TKA implants is postoperative alignment. ${ }^{1}$

Numerous studies in both the general surgical ${ }^{2-6}$ and orthopedic $^{7}$ literature show that the surgeon's handedness can affect surgical performance. Of these studies, only one focused directly on TKA outcomes related to handedness; Mehta and Lotke found that when a right-handed surgeon performed a right TKA, patients had significantly better extension, Knee Society function scores and Knee
Society pain scores one year after surgery than patients who had left TKAs performed by the same right-handed surgeon. ${ }^{8}$

To date, no study has assessed the effect of a surgeon's hand or ocular dominance on TKA alignment. Therefore, the purpose of this study was to evaluate the effect of a surgeon's hand and ocular dominance on coronal plane alignment in TKA. We hypothesized that right-handed surgeons would achieve better alignment accuracy in a right TKA compared to a left TKA. We further hypothesized that right-handed surgeons with crossed hand-ocular dominance would align a left TKA more accurately than those right-handed surgeons who have uncrossed hand-ocular dominance.

\section{METHODS}

After institutional review board approval, a retrospective chart and radiographic review was performed to evaluate the impact of the surgeon's ocular and hand dominance on postoperative alignment in 138 patients who underwent primary bilateral TKAs. All surgeries were done between 2006-2014 at the London Health Sciences Centre (London, ON, Canada). These patients had both knees replaced by the same surgeon, received the Genesis II (Smith \& Nephew, Memphis, TN) posterior-stabilized knee implant, and had standard postoperative knee radiographs available for review.

The preoperative primary diagnoses were osteoarthritis and inflammatory arthritis. Three right-hand-dominant arthroplasty surgeons, one of whom had crossed hand-ocular dominance, performed all of the knee replacements using a medial parapatellar surgical approach. The surgeons implanted all knees using a measured resection technique and aimed for a neutral mechanical axis.

To determine ocular dominance, the three surgeons performed a variant of the Porta test, ${ }^{9}$ where each surgeon extended one arm and aligned the pointer finger of the extended hand vertically with the corner of the room, with both eyes open. The surgeons then alternated closing one eye at a time and reported which eye closure caused the least alignment change. The dominant eye was recorded as the eye that, when open, saw less or no change in the alignment of the finger with the vertical corner. Surgeons were classified into two groups based on their ocular and self-reported hand dominance: the uncrossed hand-ocular group (dominance in right eye and right hand) included two surgeons and the crossed hand-ocular dominant group (dominance in left eye and right hand) included one surgeon.

Postoperative radiographs were performed using a routine protocol within two months of the surgery. Each radiograph was then reviewed and the anatomical tibiofemoral angle (TFA) was measured using the technique originally described by Hinman, ${ }^{10}$ with adjustments made for the loss of bony architecture post TKA. ${ }^{11}$ 
Anatomical TFA was defined as the angle formed by two lines originating from points bisecting the center of the femur and tibia, to the center of the tibial spines. The origin of these lines was $10 \mathrm{~cm}$ from the knee-joint surfaces.

To determine whether total knee arthroplasty alignment is more accurate on the side of the surgeon's dominant hand, we compared right and left TFA for each patient. Accuracy was defined as how close the corrected TFA was to the goal of $5^{\circ}$. Patient radiographic alignment data was expressed as the mean and standard deviation for the right and left knee, and the angles obtained for each knee were compared using a paired t-test.

Paired t-test was also used to determine if the surgeon with crossed hand-ocular dominance implanted knees more accurately on the side of their nondominant hand compared to the two surgeons who were uncrossed.

A sub-analysis using paired t-test was done on 92 patients to compare Knee Society function and pain scores of their right and left knees; this information was retrieved from hospital records of the patients' most recent follow-up.

\section{RESULTS}

A total of 138 patients were screened for eligibility. Four patients who had prior major knee trauma or open-knee surgery were excluded, leaving 134 patients in the analysis ( 77 female, 57 male). There was a significantly higher TFA with left TKAs (mean [SD], $\left.4.6^{\circ}\left[2.8^{\circ}\right]\right)$ compared to right TKAs $\left(3.8^{\circ}\left[2.5^{\circ}\right]\right)(\mathrm{P}=0.003)$. In surgeons with uncrossed hand-ocular dominance, there was a significant difference in their ability to perform an accurately aligned left TKA $\left(4.5^{\circ}\left[2.9^{\circ}\right]\right)$ compared to a right TKA $\left(3.8^{\circ}\left[2.5^{\circ}\right]\right)(\mathrm{P}=0.03)$. The surgeon with crossed hand-ocular dominance did not have any difference in accuracy between left knees $\left(4.8^{\circ}\left[2.6^{\circ}\right]\right)$ and right knees $\left(3.8^{\circ}\left[2.7^{\circ}\right]\right)(\mathrm{P}=0.07)$. When looking only at left knees, there was no difference between surgeons with crossed and uncrossed hand-ocular dominance $(\mathrm{P}=0.6)$. There was no significant difference when comparing right (82 [2.3]) and left (81 [2.4]) Knee Society function scores $(\mathrm{P}=0.09)$ and right $(47[0.7])$ and left $(47[0.5])$ Knee Society pain scores $(\mathrm{P}=0.86)$.

\section{DISCUSSION}

One modifiable variable that contributes to the longevity of TKA implants is postoperative alignment. ${ }^{1}$ The handedness and ocular dominance of a surgeon may contribute to their ability to achieve mechanical alignment. Mehta and Lotke found that, one year after surgery, patients who had a right TKA performed by a right-handed surgeon had significantly better extension and Knee Society function and pain scores than patients who had left TKA performed by the same right-handed surgeon. ${ }^{8}$ The results of this present study, however, did not agree with these findings. We did not find a significant difference when comparing right and left Knee Society function and pain scores on patients who had sequential bilateral TKAs performed by the same right-handed surgeons. Our study had the benefit of comparing bilateral knees in the same patient, therefore controlling for patient variables that may have contributed to the difference reported by Mehta and Lotke.

To our knowledge, no previous study has investigated the effect of a surgeon's hand or ocular dominance on TKA alignment. The results of this study suggests that handedness alone affects the coronal alignment of TKAs, as the TFA on left TKAs was closer to the objective of $5^{\circ}$ compared to right TKAs. These findings are in contrast to our hypothesis that right-handed surgeons would achieve better alignment accuracy in a right TKA. A previous randomized control study showed a correlation between accurate mechanical alignment after TKA and both functional and quality-of-life patient outcomes. Therefore, further research into the effect of handedness on TKA alignment, patient function, and quality of life is necessary to better understand how handedness may affect TKA results.

In this study, surgeons with uncrossed hand-ocular dominance could align a left TKA significantly more accurately than a right TKA. Although this difference is statistically significant, this needs to be interpreted with caution given that the surgeon with crossed hand-ocular dominance also performed left TKAs better then right TKAs. When looking at left knees alone, ocular dominance did not have any effect on accuracy. Further investigation into this topic is needed to understand the effects of hand and ocular dominance.

One reason we did not see a larger difference in the correlation of alignment to hand-ocular dominance may be that all of the surgeons in the study were experienced. A novice surgeon may not have this benefit, and it therefore would be interesting to repeat this study using novice surgeons, as well as those in training. Studies of this nature may lead to certain training improvements if we can better understand how hand and ocular dominance impacts learning in surgery. One limitation of our study is that it is a retrospective review with all right-handed surgeons. As such, the study could be improved by doing a prospective study using the same cohort of patients with left-handed surgeons of both crossed and uncrossed hand-ocular dominance. A second limitation of this study was the use of standard knee radiographs rather than weight-bearing fulllength radiographs. However, previous studies have shown that standard knee radiographs are a reliable method for measuring anatomical TFA as a proxy for mechanical axis; ${ }^{10-13}$ the physiologic valgus offset for the anatomic axis has been demonstrated to range from $4^{\circ}$ to $6^{\circ}$. Consequently, we used a TFA of $5^{\circ}$ as a proxy for a neutrally aligned mechanical axis,,$^{12,14-15}$ which therefore resulted in the left knees being more accurately aligned.

In summary, hand dominance has previously been demonstrated to affect surgical performance on side-specific procedures, as also demonstrated in this study. Although demonstrated to have an effect in other areas of performance, ocular dominance had not been previously examined in surgical performance. Ocular dominance did not affect TKA alignment in the coronal plane. Further research is necessary to confidently say that ocular dominance has no effect on TKA outcomes. 


\section{REFERENCES}

1. Jeffery RS, Morris RW, Denham RA. Coronal alignment after total knee replacement. J Bone Joint Surg Br. 1991 Sept; 73(5): 709-14.

2. Grantcharov TP, Bardram L, Funch-Jensen P, Et al. Impact of hand dominance, gender, and experience with computer games on performance in virtual reality laparoscopy. Surg Endosc. 2003 Jul; 17(7): 1082-5.

3. Power TW, Bentrem DJ, Nagle AP, Et al. Hand dominance and performance in a laparoscopic skills curriculum. Surg Endosc. 2005 May; 19(5): 673-7.

4. Hanna GB, Drew T, Clinch P, Et al. Psychomotor skills for endoscopic manipulations: differing abilities between right and left handed individuals. Ann Surg. 1997 Mar; 225(3): 333-8.

5. Pouw L, Tulloh B. Laparoscopic cholecystectomy for the left-handed surgeon. Br J Surg. 1995 Jan; 82(1): 138.

6. Schueneman AL, Pickleman J, Freeark RJ. Age, gender, lateral dominance, and prediction of operative skill among general surgery residents. Surgery. 1985 Sep; 98(3): 506-15.

7. Moloney D, Bishay M, Ivory J, Et al. Failure of the sliding hip screw in the treatment of femoral neck fractures: "left-handed surgeons for left-sided hips. Injury. 1994; 25(Suppl 2): B9-13.

8. Mehta S, Lotke PA. lmpact of surgeon handedness and laterality on outcomes of total knee arthroplasties: should right-handed surgeons do only right TKAs? Am J Orthop. 2007 Oct; 36(10): 530-3.

9. Roth LH, Lora AN, Heilman KM. Effects of monocular viewing and eye dominance on spatial attention. Brain. 2002 Sep; 5(Pt 9): 2023-35

10. Hinman RS, May RL, Crossley KM. Is there an alternative to the fullleg radiograph for determining knee joint alignment in osteoarthritis? Arthritis Rheum. 2006 Apr 15; 55(2): 306-13.

11. Chang CB, Choi J-Y, Koh IJ, Et al. What should be considered in using standard knee radiographs to estimate mechanical alignment of the knee? Osteoarthritis and Cartilage. 2010 Apr; 18(4): 530-8.

12. Kraus VB, Vail TP, Worrell T, Et al. A comparative assessment of alignment angle of the knee by radiographic and physical examination methods. Arthritis Rheum. 2005 Jun; 52(6): 1730-5.

13. Colebatch AN, Hart DJ, Zhai G, Et al. Effective measurement of knee alignment using AP knee radiographs. Knee. 2009 Jan; 16(1): 42-5.

14. Hsu RWW, Himeno S, Coventry MB, Et al. Normal Axial Alignment of the Lower Extremity and Load-Bearing Distribution at the Knee. Clin Orthop Relat Res. 1990 Jun; 255: 215-27.

15. Moreland JR, Bassett LW, Hanker GJ. Radiographic analysis of the axial alignment of the lower extremity. J Bone Joint Surg Am. 1987 Jun; 69(5): 745-49.

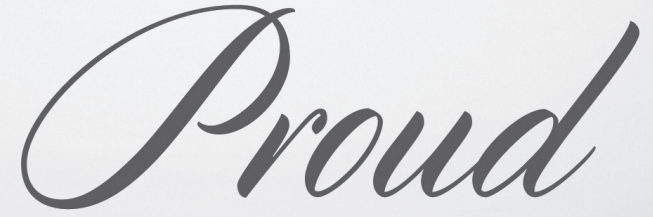

to be a part of your medical journey

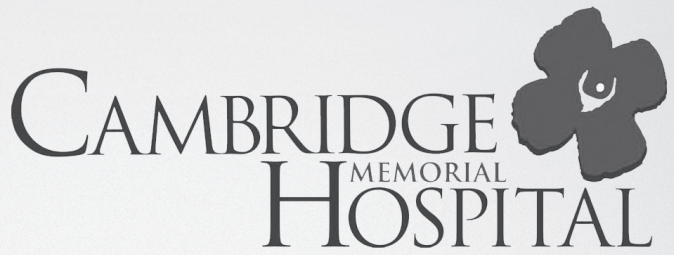

